\title{
Extensible Statistical Software: On a Voyage to Oberon
}

\author{
Günther Sawitzki \\ StatLab Heidelberg \\ Im Neuenheimer Feld 294 \\ D 69120 Heidelberg
}

\begin{abstract}
Recent changes in software technology have opened new possibilities for statistical computing. Conditions for creating efficient and reliable extensible systems have been largely improved by programming languages and systems which provide dynamic loading and type-safety across module boundaries, even at run time. We introduce Voyager, an extensible data analysis system based on Oberon, which tries to exploit some of these possibilities.
\end{abstract}

Keywords. Statistical Software, Software Portability, Extensibility, Object-Oriented Programming

\section{Introduction}

Software technology has changed over the last years, and recent changes have opened new possibilities for statistical computing. In particular, the conditions for creating efficient and reliable extensible systems have been largely improved. A basis has been established to work with software components. Main improvements come from research related to Project Oberon at ETH Zürich [Wirth \& Gutknecht 1992]. To explore how these possibilities can be made fruitful in statistical computing, we have designed and implemented Voyager ${ }^{1}$, an extensible data analysis system [Sawitzki, 1994].

Software technology is moving from self-contained applications and programs towards software components. The challenge is to design modules for code-reuse, to define adequate abstract data types, and to give a basis of extensible types and objects. We will give some examples how these challenges are met in Voyager.

The Voyager project has been driven by the need to define the directions of our own computing environment for the years to come. Although we have been guided by the specific needs at StatLab Heidelberg, the critical requirements seem to be quite common. In the view of a rapidly changing technical environment, we need portable statistical systems which do not depend on specific hardware or software environments. Statistical research over the last years has shown the potential contribution of resampling and other data based methods. To make use of this contribution, data analysis and resampling/simulation facilities must be closely integrated. Finally, as a research institution, a main focus is on the development and application of new methods. This asks for extensible software allowing to integrate results from recent research. None of these three requirements is easy to fulfill. Porting software may be a pain when old design scars break up in new wounds, and integrating analysis and simulation may need careful considerations. But at present, the major challenge among these requirements is true extensibility.

To start with the first problem, portability: our solution was to build on a portable operating system. After careful consideration, the system and language of choice was Oberon. We will give a very short

1 voyager n. person who makes a voyage (esp. of those who, in former times, explored unknown seas).

-1 -

30. Oct. 1995. Submitted to the Journal of Computational and Graphical Statistics

WWW entry point: http://statlab.uni-heidelberg.de. Comments to gs@ statlab.uni-heidelberg.de, please. 
impression of the Oberon system in the next section. Then we show how Voyager, our system, is built upon Oberon, and show some basic components of Voyager. Finally we discuss how extensibility is achieved by Voyager making use of the Oberon environment. But before proceeding, we have to take a closer look at some problems related extensibility.

\section{Extensibility}

There are several reasons to ask for extensibility. For example, your software may do nearly the right thing, and you just want to add the missing bit. Or it does the right thing, and you want to wrap it up to provide easier handling and integration into reports. Or, as usual in our case, you want to add new functionalities which make use of the services already provided. Several methods are available to provide extensibility. If your system is a closed system, you have to modify the code and recompile it - there are no other possibilities in a closed system. You may get a similar effect by controlling your system by a script - be it a script for some macro language integrated with the system, or controlled by an external script system. Or your system may allow for external functions and back calls. If the requirements are modest, these means may be sufficient. If you want to use typical tools of data analysis, like interactive graphical methods, these means may fall short: for interactive graphics, you need a tight feedback loop from user input to system response. Timing behaviour may become critical. Other methods come into play, like message passing or process-to-process communication. These methods however require that your system is open for communication.

A key requirement is that extensibility should not compromise system stability. Guaranteeing for stability cannot be based on good hopes, but must be built into the system. Consistency must be assured even for non-anticipated extensions, and compatibility of versions must be checked. Extensions may be added at any time, even long after the design of the original systems, and by any user, not necessarily informed about all assumptions of the original implementer. It must be guaranteed that memory usage and parameter conventions of the extension and the original program coincide, and that any mismatches are detected. There are well known techniques to help maintaining system stability, such as modular design, control of import/export, type checking. The common questions of maintainability and portability reoccur in a more demanding form for extensible systems.

\section{Strategy: Modularity and Separation of Concerns}

Modular programming is a basic strategy for the development of stable programs. A module is a program unit with a well defined function, communicating with other program units only via well defined inputs and outputs. To achieve modular programming, you must adhere to a modular programming style, and you need a language and programming environment which support working with modules and can shield the internal information of a module from external access.

Modularity is a formal concept. It must be accompanied by a strategy how to decide upon the decomposition into modules or components. A leading idea is separation of concerns. Each component should fulfil the task it is designed to do, and it should withhold from other tasks. Defining the proper concerns is the first step, and the design of corresponding components or modules has to follow.

Separation of concerns is a general principle. It should hold for all components of the statistical appli-

$$
-2 \text { - }
$$

30. Oct. 1995. Submitted to the Journal of Computational and Graphical Statistics

WWW entry point: http://statlab.uni-heidelberg.de. Comments to gs@ statlab.uni-heidelberg.de, please. 
cation, but also for the relation between statistical application and the embedding system programs. Defining the proper concerns often goes hand in hand with identifying a certain level, or layer responsible for some task.

As an example, memory management is a common task, and garbage collection - the identification and release of no longer used computing resources - is a necessary, but most annoying aspect of this. A decision has to be taken who is responsible for garbage collection. You cannot identify all clients of objects if the system continues to "grow" and clients have their own memory management. So to maintain system stability, you either can keep garbage collection on the application level, implying that you deny the rights to do memory management to the extension - an unrealistic restriction -, or you need a system wide garbage collection. We prefer an operating or run time system to handle it. We try to be cooperative and arrange our memory allocation in a way which makes garbage collection easier. But no computational statistician should be required to spend time on these things which would be better handled by computational scientists - garbage collection should be a system service, not handled by the application or extensions.

\section{Oberon}

The Oberon operating system [Reiser 1991, Wirth and Gutknecht, 1992], and the Oberon language [Wirth 1988, Reiser and Wirth 1992, Wirth and Mössenböck, 1993], have been developed at ETH Zürich. Both are intimately related: The Oberon operating system is written in Oberon, and many Oberon language constructs find most convincing applications in the Oberon operating system. Oberon is a single tasking operating system. It does allow for background tasks, but the usual processes are executions of a single command and return to a common base level as soon as the command is executed. Garbage collection is provided by the Oberon system.

The Oberon operating system is extremely economical. A basic Oberon system takes about $300 \mathrm{~K}$ (without extensions, of course; extensions can add an arbitrary amount). This count includes a compiler and an editor. As a consequence, you can implement a full Oberon operating system on top of another operating system, with full functionality, and still use less resources than some of todays editor extensions would use [Franz 1993]. The designed economy is one of the bases for Oberon's portability. The Oberon operating system is available as a native operating system (on the Ceres workstations or IBM PCs, for example). But what is more interesting is that it is available as an emulation on top of most of the common operating systems (UNIX, MS-Dos, MS-Dos\&Windows, LINUX, MacOS etc.) and for most of the common hardware architectures (Intel x86, Motorola 68xxx, PowerPC, MIPS Risc, SPARC etc.). As with all evolving systems, variants of Oberon do exist. Unless stated otherwise, we will use Oberon in the sense of Oberon operating system Version 4. This is a deliberate decision. Other variants (Oberon System 3, Oberon/F) offer interesting perspectives. The System 3 variant introduces support of persistent objects and libraries as a system service [Gutknecht 1993, Marais 1994]. Augmented by the Gadgets system [Marais 1995] it offers a desktop model and a document oriented interface. Oberon/F is a cross-platform development system based on Oberon providing a platform-specific look-and-feel and

$$
-3-
$$

30. Oct. 1995. Submitted to the Journal of Computational and Graphical Statistics

WWW entry point: http://statlab.uni-heidelberg.de. Comments to gs@ statlab.uni-heidelberg.de, please. 
heading towards an integrated document model [Pfister et al., 1994]. But at present Oberon version 4 is the base version if you consider portability over a wide range of platforms. It is implemented on most platforms, and all critical components are available in source form.

The Oberon language is a strongly typed language in the tradition of Algol and Pascal. In comparison to Pascal (or Modula), Oberon is cleaned-up and streamlined. Some features have been removed or modified to allow for an efficient compilation, others have been added, mostly to increase the safety of the language, and support for object oriented programming is integrated.

Oberon allows modular programming with export control on module level. It encourages a very fine grained modularity. Type safety is guaranteed across module boundaries and includes type safety for run time extensions. Once loaded, modules stay resident and their status is preserved unless the module is explicitly unloaded. Memory is shared, and access to variables is controlled on a per module basis. The definition of a module contains a description of exported data structures and permissions to access variables. A calling module explicitly imports the called module. Since the definition of exported data structures is preserved during compilation, consistency can be checked without need to access the source code. Moreover, the compiled code contains keys which can help to identify version mismatches at load/run time.

This contrasts drastically with the situation you would encounter in $\mathrm{C} / \mathrm{C}++$, say. With $\mathrm{C} / \mathrm{C}++$, you can access any memory cell once you have an appropriate starting pointer - there is no true modularity with $\mathrm{C} / \mathrm{C}++$. And with $\mathrm{C} / \mathrm{C}++$, you, the programmer, are responsible that header files, source files, compiled object code and libraries are synchronized. If you allow for call-back functions, you are responsible to take all consistency checks. You may use tools to help you, but there is no compile-time or run time consistency check in $\mathrm{C} / \mathrm{C}++$, while type safety (and module version consistency) is supported by Oberon even at run time.

There is no separation between system and application programs in Oberon. In particular, all system modules are accessible as a free library of shared components. The Oberon system includes support for high level abstractions. For example, Oberon has an abstract data type Text, which knows about attributes (like colour and style) and has methods like append or insert, write to file, read from file. Moreover, the Text data type allows for "text elements" which may be represented by anything, not only letters. Active programmed elements can be an integral part of an Oberon Text, flowing with the text and modifiable by the same methods as any other components of the text (for example a "life" clock can be imbedded in a running text). A graphical display system is an integral part of Oberon. By default, he Oberon system includes a tiled graphical window system.

The original design of Oberon tries to avoid modes. By convention, text fragments of the form M.P are considered commands. They can be executed and yield a call to procedure $\mathrm{P}$ in Module M. Execution is associated to one of the mouse buttons. Any text in any display is active. It may be input text entered by the user, or output of previous commands, or any text accessible to Oberon. In the basic Oberon system, text is presented in frames and can be selected using a mouse. Thus commands may be executed by entering commands in any display frame, or by activating any command which may be visible in the display frames. There is no special command line or menu bar. Menus are only special in that they have other attributes, showing text in inverted colours. In all other respects they are display frames like any 
other frame and are under full control of the user. In particular, the user can add or remove entries in menus like in any other text, and can execute commands in menus like in any other position.

\section{Voyager}

Voyager adds a layer on top of the general Oberon system. The task of Voyager is to provide the basis for statistical computing in an Oberon environment. It provides data management, proper statistical computing, and display services. Of course as far as the proper statistical computing component is concerned Voyager like any other statistical program, draws upon the usual resources, such as BLAS/LAPACK etc. for linear algebra, or the Applied Statistics series and other commonly available sources for statistical algorithms.

The display components have to go own ways. To guarantee the usual interactions of data analysis, like selection, brushing, slicing [Becker et al. 1987] in a portable way, you need a graphic environment which is prepared for interaction. The Voyager display system supports these actions. Following the principle of separation of concerns, Voyager restricts itself to the proper task of statistical graphics. Instead of providing one more presentation system or one more application system, Voyager provides components which can be used even with previously existing application programs - like some editor of the user's choice. This works using the usual message passing mechanism of Oberon [Marais 1995]. The Voyager graphics are provided as specific text elements, which, as mentioned before, can be part of any text. When seen by an editor, Voyager graphics are just some text elements flowing with the text like letters or other elements. As long as any of the usual text actions (insert, cut, paste etc.) is required, it is handled by the editor. If the editor encounters any action message it cannot handle and if the input is focussed on a text element, a corresponding message is forwarded to the text element to take the appropriate action. If the text element is one of Voyager's displays, Voyager takes over. This message passing mechanism allows Voyager to respond and provide dynamic plots.

By convention, Voyager output takes a form which can be used as Voyager input again. Of course it need not be typed in again - following the Oberon conventions, all commands allow references to text selections either by redirecting input (using an arrow character) or by imbedding it in a command (using "@” as a reference mark). So the output of a regression yields a regression equation which can be used as input to specify a model, which in turn can be used to draw new samples, thus allowing the required close integration of data analysis and resampling or simulation. [Figure 1]

-5 -

30. Oct. 1995. Submitted to the Journal of Computational and Graphical Statistics WWW entry point: http://statlab.uni-heidelberg.de. Comments to gs@ statlab.uni-heidelberg.de, please. 
Voyger.ReadData Examplex8.2 4.41.31.82.95.2\%

Voyger.ReadData Exampley $7.24 .91 .81 .01 .96 .2 \mathrm{~N}$

Examplex: 6 numeric values read.

mirr: 1.3

max: 8.2

Examplet: 6 mumperic ralues read.

mirr: 1.0

max: 7.2

Voyger.Regress Exampley Examplex $\infty$

Example' $=0.9596$ ExampleX $+0.0270+$ err ; $\quad$ varierrí $=0.9950$
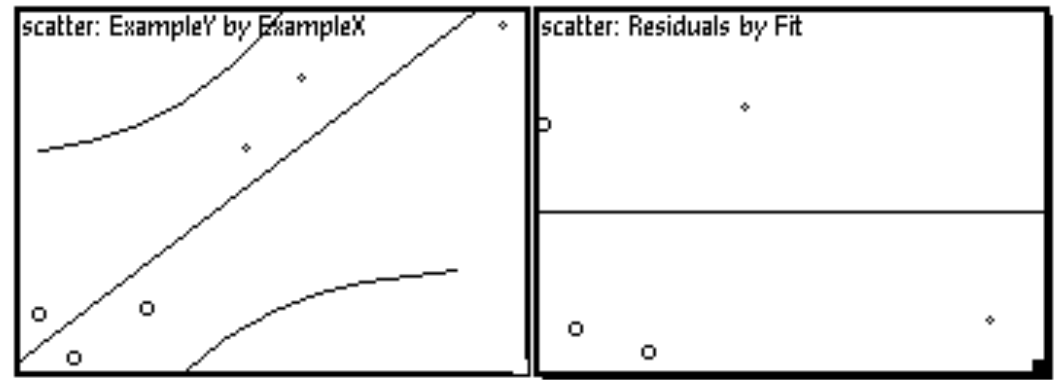

Voyger Model $\uparrow$

Voyager.Resample $n=50$ samples=1000 $\omega$

1000 random samples drawn from recent regtession model
Welcorme to the Final Rielease of ivacoberon w4/ mf

'voyager.LaLnhch

Woyager Mrólat

Woyager. Base 0.1a1

Woyger.Name 01 191

\section{System.Tod | Clase Coun Grow Serch Stare}

Edit.Oper1 $\uparrow$

Edit.Frint Duick:Draw *

System.5howhodules

System. ShowCommands $t$

Systern.5tate $t$

Compiler.Compile *

Compiler.Compile $\% / s$

EjgRer Smaller 14

System. Fecall

Edit.Fiecall

inst closed merer

System.Cirectary $t$

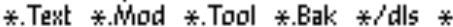

\begin{tabular}{|l|}
\hline Application: Oberon Edit \\
\hline Voyager: version 0.a1 \\
\hline Oberon: MacOberon 4.1F \\
\hline Host System: MacOS System 7 \\
\hline
\end{tabular}

Figure 1. Integration of Voyager in a layered architecture. Voyager elements in an editor environment. By convention, the Oberon screen is divided into tracks. The track on the left shows a default Oberon editor window with Voyager in- and output: graphics are imbedded as text elements. Interaction, like brushing, is handled by these text elements, whereas in all other aspects they behave like any other text element or character.

Building upon the generic Oberon graphic system, Voyager provides the necessary graphical basis for data analysis. One- two and tree dimensional plots, including rotating 3d-plots, are included in Voyager. All plots can be linked and support the usual action such as brushing, identification etc. [Figure 2]

-6 -

30. Oct. 1995. Submitted to the Journal of Computational and Graphical Statistics

WWW entry point: http://statlab.uni-heidelberg.de. Comments to gs@ statlab.uni-heidelberg.de, please. 


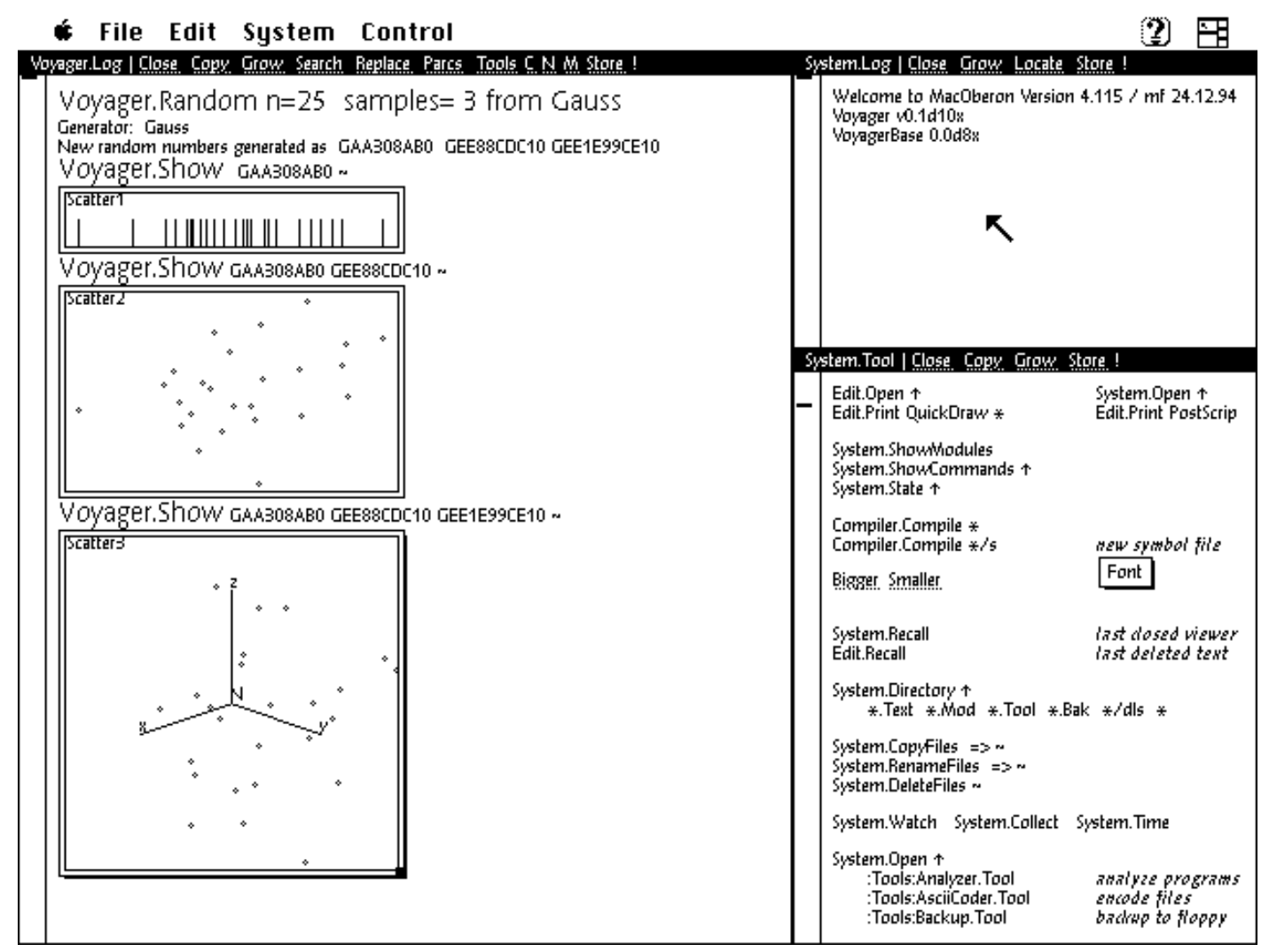

Figure 2. Basic Graphics in Voyager. One- two and tree dimensional graphics are supported, including rotating scatter plots. By default, the corresponding plot is selected based on the number of parameters of Voyager.Show. All displays support brushing and can be linked.

Of course scatter plots are not the only displays provided by Voyager. For one dimensional data, for example, the basic displays comprise the usual collection of scatter plots, histograms, empirical probability functions, box\&whisker plots and shorth plots. If you ask Voyager to show the data, it will respond with a default plot. This default may depend on additional information, such as the number of variables you want to see. If you prefer a different plot, you have to change the default setting, or you have to chose a different plot explicitly. [Figure 3]

-7 -

30. Oct. 1995. Submitted to the Journal of Computational and Graphical Statistics

WWW entry point: http://statlab.uni-heidelberg.de. Comments to gs@ statlab.uni-heidelberg.de, please. 


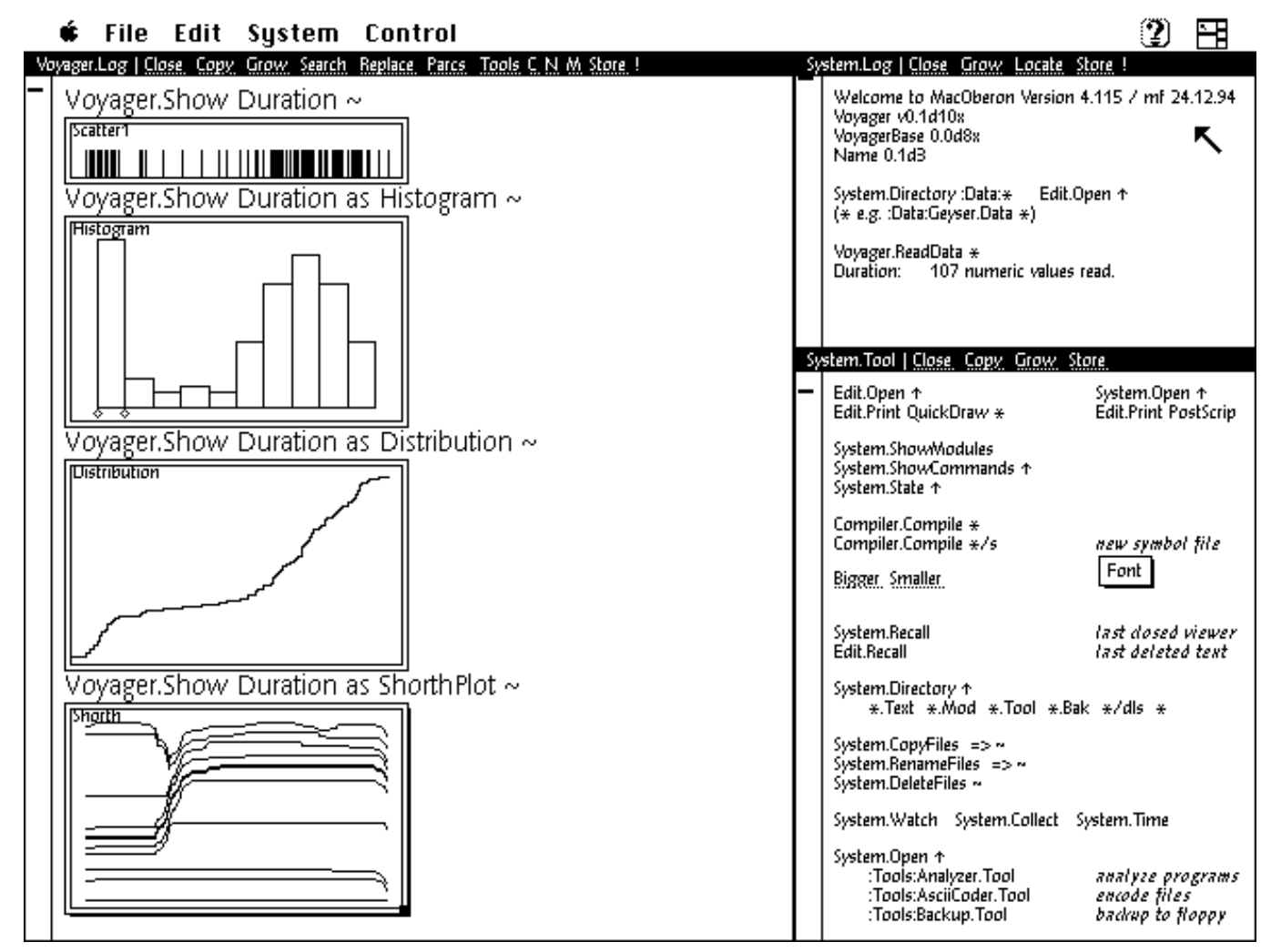

Figure 3. More Graphics in Voyager. Some more basic displays for one dimensional data in Voyager. Interactive facilities are supported by all displays where appropriate, with display specific extensions (e.g. hot spots on the histogram allow to move the histogram offset or bin width by sliding one of the hot spots below the base line).

\section{The Voyager Design}

Voyager tries to separate issues of user interface and presentation from statistical computing. We have seen that Voyager provides graphical displays as text elements which can be imbedded in a text and presented by any Oberon text editor, for example. Since it is a common feature in Oberon to be able to respond to "alien" interactions and forward them to the extensions if necessary, interactivity can be preserved for the embedded elements. The embedded graphics can stay alive and respond to additional interactions without any need of changes to the embedding editor.

This is exploited in Voyager support a basic prerequisite for many techniques of interactive data analysis: selections and linked windows. We have seen an example in the regression plot shown above. The scatter plot and the residual plot are linked. Brushing over one of the plots defines a selection, highlighted by circles in that example. The selection is shared by all linked plots and highlighted correspondingly. This linking of displays, or any objects, is a basic feature of Voyager. To see how it is realized, we must take a look of the design of Voyager.

Voyager consists essentially of six components: a display system and a command system, which form the most visible part of Voyager, a data management system, a computing system containing essentially

$$
-8-
$$

30. Oct. 1995. Submitted to the Journal of Computational and Graphical Statistics

WWW entry point: http://statlab.uni-heidelberg.de. Comments to gs@ statlab.uni-heidelberg.de, please. 
statistical algorithms and transformations, a base system responsible for abstract management, and a general toolbox.[Figure 4]
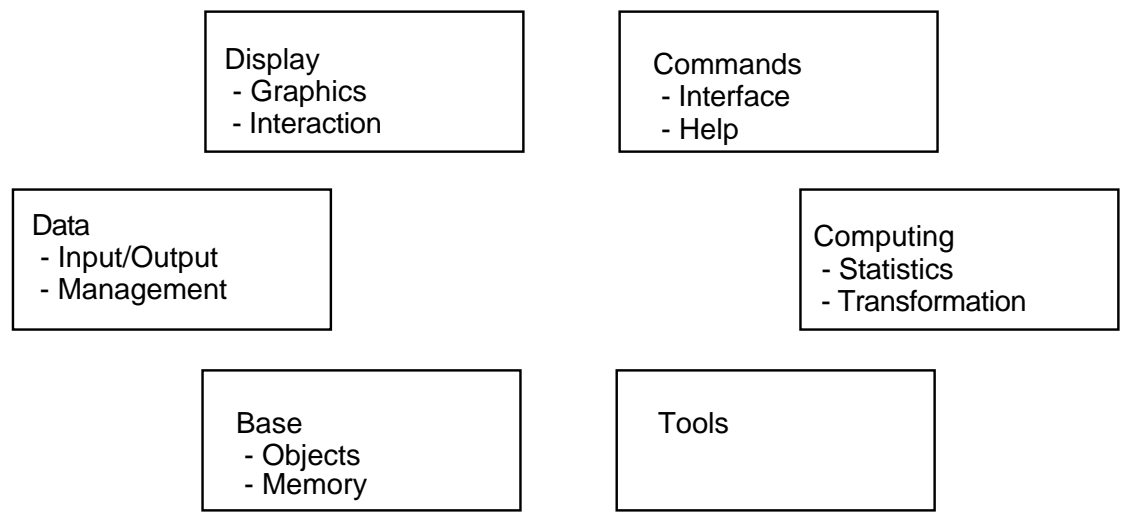

Figure 4. Basic Voyager components

These components do not form a hierarchy, but are on the same level. Any one component can make use of services of other components as it might be necessary. In particular, any component can make use of the services of the base component. The Voyager base is responsible for object management. At this level, Voyager objects are abstract data types. No assumption is made about the interpretation of these data, but a well defined set of actions is supported in a generic way. One action for an object is to register with the base: this makes the object known to the Voyager base, which in turn may generate necessary administrative information (such as an identification or a time stamp). Another action for an object is to subscribe as a client to some other object. Whenever that object changes, the Voyager base guarantees that an update message is sent to all subscribers, which on their turn have to handle the update message. And this is all that is behind brushing and linked windows: the window displays subscribe as clients to one common object, the current selection. Brushing changes the selection. In response, the Voyager base sends an update message to all subscribers which adjust their appearance accordingly. [Figure 5]

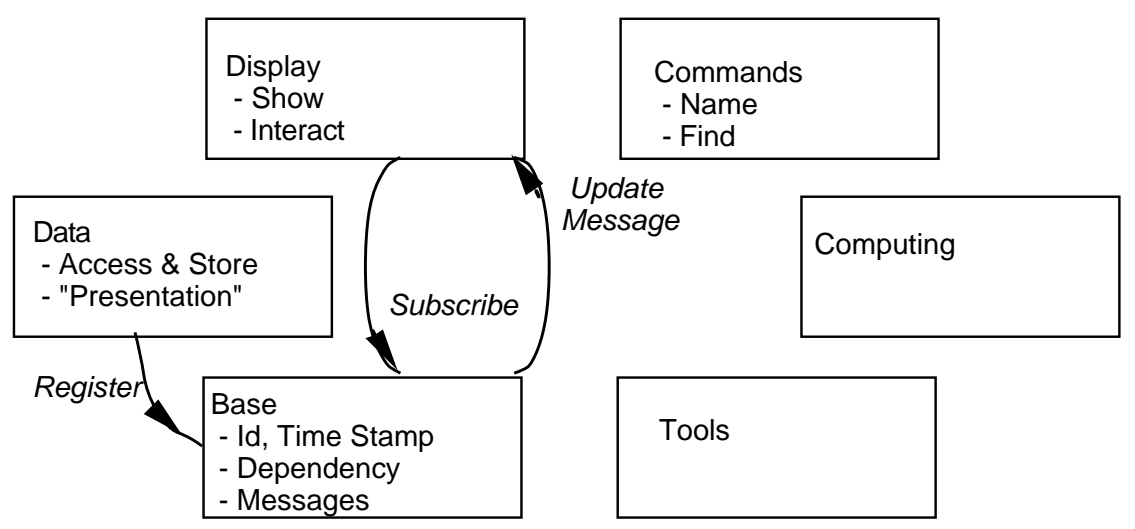

Figure 5. Object and Message Management. A subscribe mechanism, together with update messages, is used to implement brushing, linked windows and other dynamic features.

For interactive data analysis, interaction and display become intimately related with statistical computing.

$$
-9 \text { - }
$$

30. Oct. 1995. Submitted to the Journal of Computational and Graphical Statistics WWW entry point: http://statlab.uni-heidelberg.de. Comments to gs@ statlab.uni-heidelberg.de, please. 
If you can truly interact with the data, each display element, including each data point in the display, potentially is a control element used for interaction. Controlling graphics by external gadgets may become a cumbersome process. The Voyager subscription mechanism provides a reliable and efficient basis for many techniques of interactive data analysis.

Since the subscription mechanism is provided as a basic mechanism available for all objects, the same mechanism can be applied for a variety of purposes. Assume, for example, that you have some random sample. You analyze it, and show the result in some plot. Now make the random sample depend on the time clock. Whenever the time clock changes (by some fixed amount) say, the random sample is told to update itself. Since drawing the previous sample had changed the seed, this gives a new sample. The change is propagated - and you have a running simulation.

\section{Look and Feel}

Oberon is shifting software technology from a technology of programs and routines to a technology of software components. Components can be exchanged and replaced individually, and with Oberon, component exchange is even possible at run time. Exchangeability does not stop at the top level: by replacing the top level, different user interfaces can be achieved, and you can get a different look and feel. Voyager provides services on an intermediate level between the basic system and the user interface. In a modular architecture, you can use these services even when the other components are exchanged.

So far, we have shown Voyager in the environment defined by Oberon V4 and its standard editor, Edit. Edit could be replaced by any other editor which can handle standard Oberon texts, since text elements and command execution are generic in Oberon. The reason for the choice of Oberon V4 is that this is the fundamental Oberon variant, available on most platforms and in source form - so portability and maintainability have a sound basis. These choices are deliberate, and other choices are possible. Voyager does not assume a specific user interface. The user interface, as all of the presentation level, is left to the open. To a large extent, the proper statistical application and the user interface can be developed independently. There are other systems which undergo more compromises with user preferences for a certain look and feel. As statisticians, we should take these aspects seriously. Statistics does not improve with an attracting user interface. But we do not only have to have the right results, but we have to bring them to perception. So we should take into account the preferences and habits of our users.

For user who prefer graphical dialogs a light weight solution is to have a dialog generate a command text, which is then passed to Voyager for execution. Since Oberon provides a standard mechanism for command calls, there is no need for any internal adjustment: Voyager can accept commands, irrespective of the source. If the user prefers dialogs instead of textual commands in an editor, the choice is free. A convenient implementation of dialogs is available [Knasmüller, 1994] for free combination. [Figure 6]

$-10-$

30. Oct. 1995. Submitted to the Journal of Computational and Graphical Statistics

WWW entry point: http://statlab.uni-heidelberg.de. Comments to gs@ statlab.uni-heidelberg.de, please. 
These are some prepared initial examples for Voyager. Output will go to the current caret position, or to the Oberon system log. To awoid corrupting this script, execute

Voyager.OpenLog temp.log

\section{temp.log | Close Copy Grow Search Replace Parcs Togls C N M Store !}

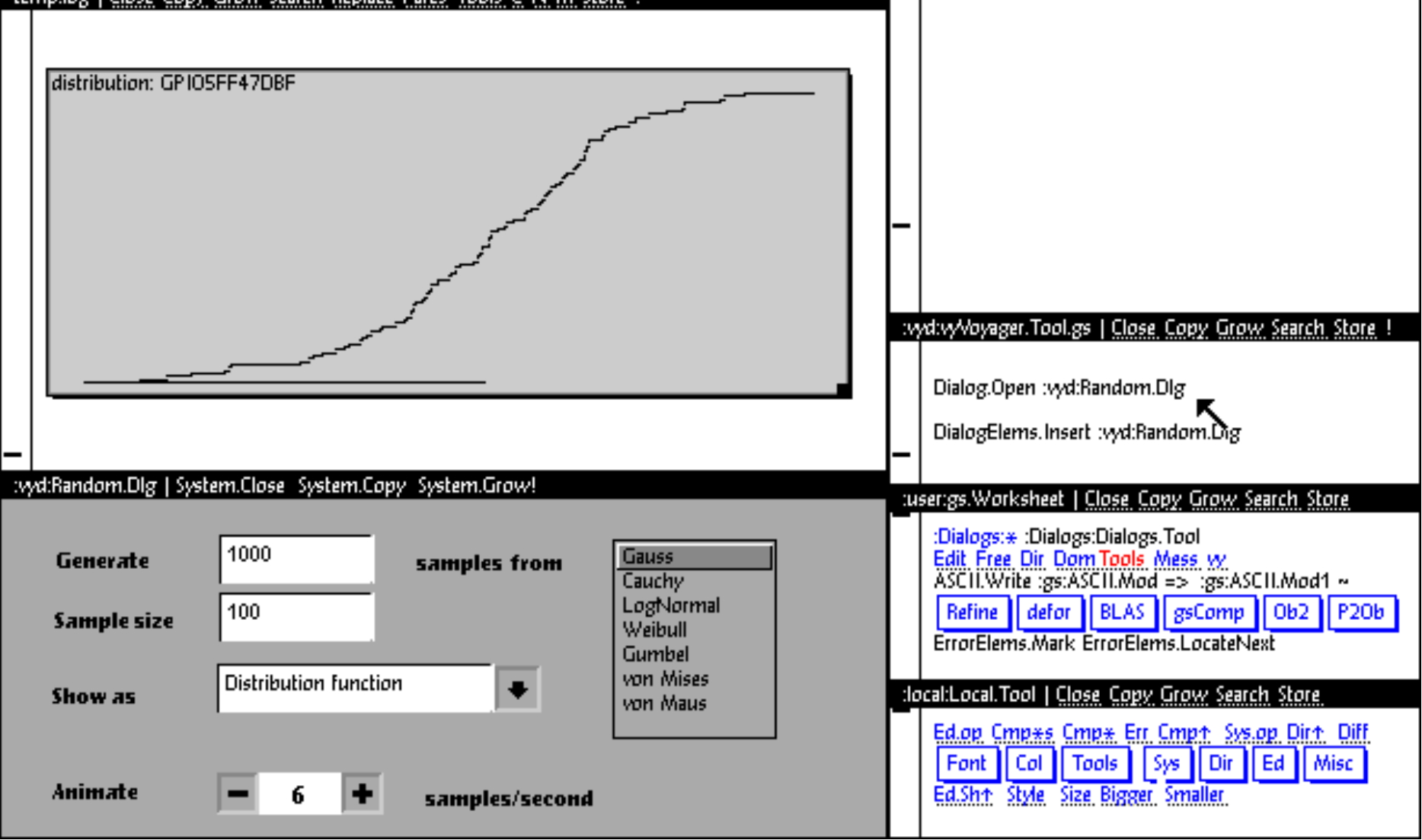

Figure 6. Voyager controlled by Dialogs. A collection of independent interface components, Dialogs [Knasmüller 1994], is used here to control a simulation in Voyager with results displayed by a standard editor.

A different possibility is to use System 3 with Gadgets. Oberon System 3 provides support for persistent objects and libraries, and Gadgets are interface components making use of Oberon System 3 to allow for a customizable user interface. Gadgets provides a collection of reusable interface elements familiar to users of Windows or similar environments. Under System 3 with Gadgets, Voyager can make use of these additional facilities to provide a different user interface. [Figure 7]

$-11-$

30. Oct. 1995. Submitted to the Journal of Computational and Graphical Statistics

WWW entry point: http://statlab.uni-heidelberg.de. Comments to gs@ statlab.uni-heidelberg.de, please. 


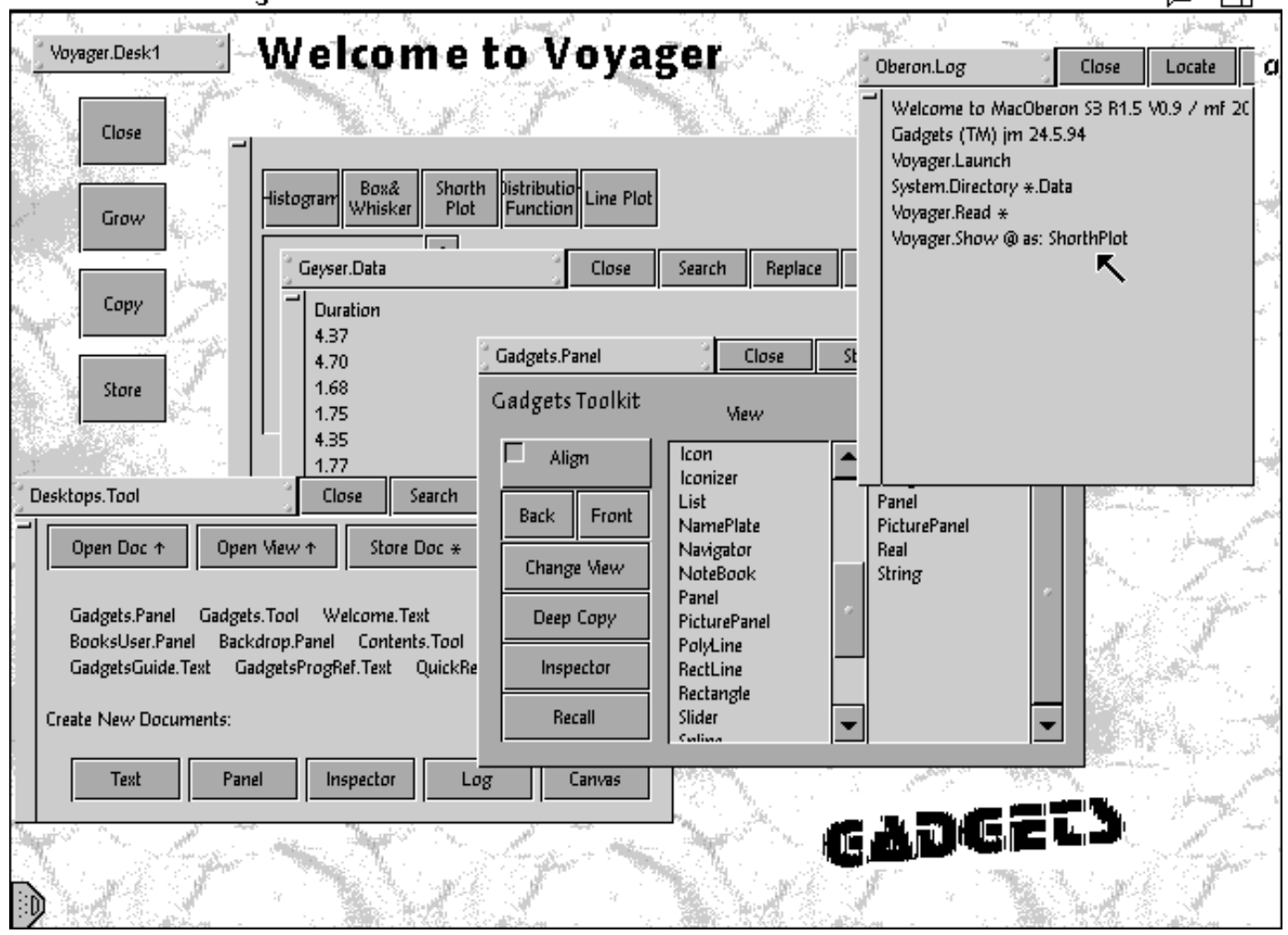

Figure 7. A Voyager prototype using Gadgets. The experimental Gadgets system provides a rich collection of reusable interface elements which can be used to present Voyager with a different user interface.

Oberon/F is another Oberon variant, providing a seamless document model as default interface. Under $\mathrm{MacOS}$, Oberon/F has the look and feel of a usual Macintosh editor, as it has the Windows look and feel under MS-Windows. Used with Oberon/F, Voyager displays appear as parts of a document. [Figure 8]

-12 -

30. Oct. 1995. Submitted to the Journal of Computational and Graphical Statistics WWW entry point: http://statlab.uni-heidelberg.de. Comments to gs@ statlab.uni-heidelberg.de, please. 


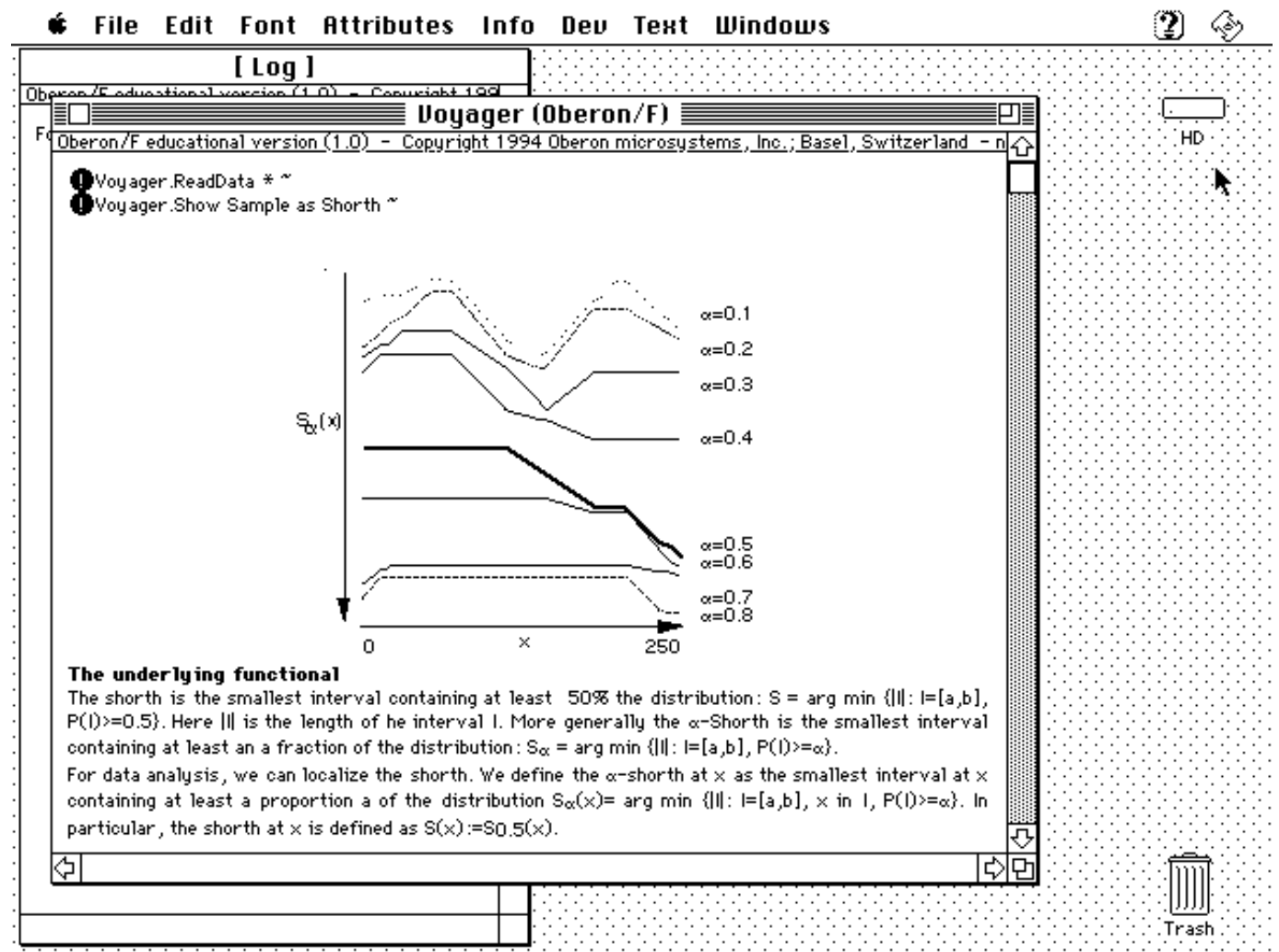

Figure 8. A Voyager prototype under Oberon/F. Oberon/F has an interface which is based on a document model, as appropriate for the home operating system. In this example, Oberon/F is running under MacOS and is using Macintosh conventions. Voyager appears in the form of a conventional Macintosh document, with the exception of some special markers to denote executable statements in the text. Voyager output is integrated seamlessly into the document, an interactive version of [Sawitzki 1994a].

We separated user interface from application programming. This is the reason why Voyager can be integrated easily into these variants of the Oberon environment. To test the design implications more thoroughly, we have implemented a variant of Voyager on a Newton PDA. The completely different information handling on the Newton ("data soups" instead of files) and the completely different user interaction (pen based instead of mouse and keyboard) was a helpful test bed to clarify the design.

[Figure 9]

-13 -

30. Oct. 1995. Submitted to the Journal of Computational and Graphical Statistics

WWW entry point: http://statlab.uni-heidelberg.de. Comments to gs@ statlab.uni-heidelberg.de, please. 


\section{Voyager}

- Soup Liste8409:SmartList1.5:PYTHIA

- Wariable F3

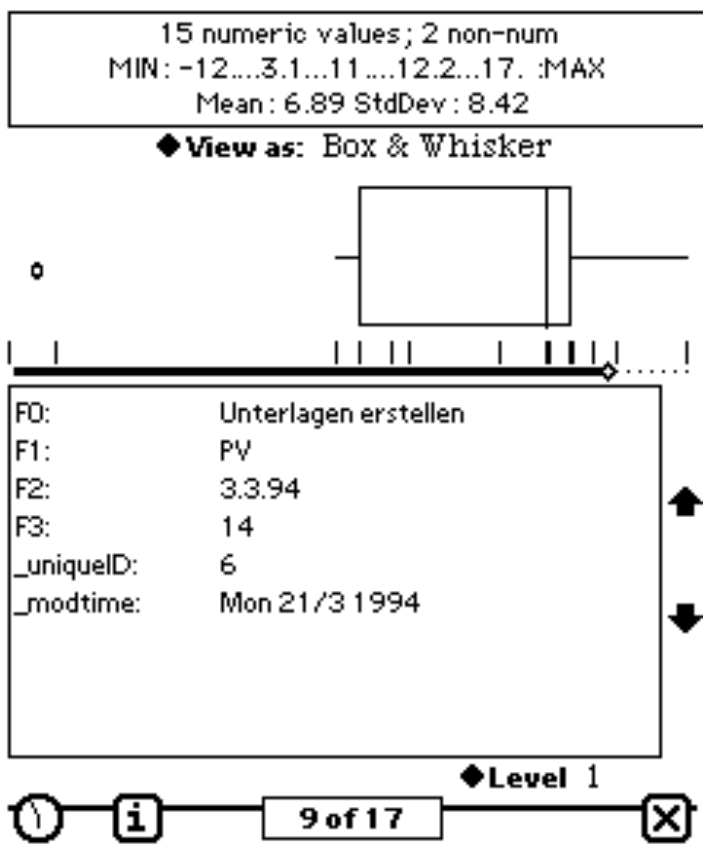

Figure 9. A Voyager prototype on a Newton PDA. An example of a different user interface on top of Voyager, and a different underlying operating system. The limited display space of a PDA and the pen based input suggested a careful grouping of output information.

\section{Extending Voyager}

All of Oberon is designed for extensibility, and any Oberon application can take benefit from this possibility. The basis for extensibility is a common message passing mechanism, and a shared address space at run time. By convention, messages are passed to a message handler which has precautions for "unexpected" messages [Marais 1995]. This allows for future extensions. To protect system stability, shared memory access is type-safe across module boundaries. These means are provided by Oberon, and it is up to any application to make use of it. Moreover, any functionality previously available can be used for extensions. If previous code has been designed for re-usability, it can be integrated as a component in new applications.

So far we have seen how Voyager is designed, how it is decomposed into components, and how it is integrated into the Oberon system. Each Voyager component can be replaced by other implementations, allowing considerable flexibility. We now turn to specific possibilities for extensibility opened in Voyager, using the Oberon background. We already mentioned that modules loaded in Oberon stay resident and their information is accessible if it is exported. If a module does not export its information, it is closed and not available for extension. If information is exported, how can we use it for extension?

-14 -

30. Oct. 1995. Submitted to the Journal of Computational and Graphical Statistics WWW entry point: http://statlab.uni-heidelberg.de. Comments to gs@ statlab.uni-heidelberg.de, please. 
As an example, let us return to regression. We have shown a simple default regression output of Voyager in Figure \#. Suppose you want to illustrate the consequences of the choice of the level in a classroom or consulting situation. Wouldn't it be nice to have a means to vary the confidence level and see how the confidence bands change? If you have the original code, of course you can change the program. Let us for the sake of the example assume that this has been written long ago by someone else, and you do not have access to the code. The standard Oberon command Browser.ShowDef lets you inspect the exported types, variables and commands of a module. For the simple linear regression module for example, you get

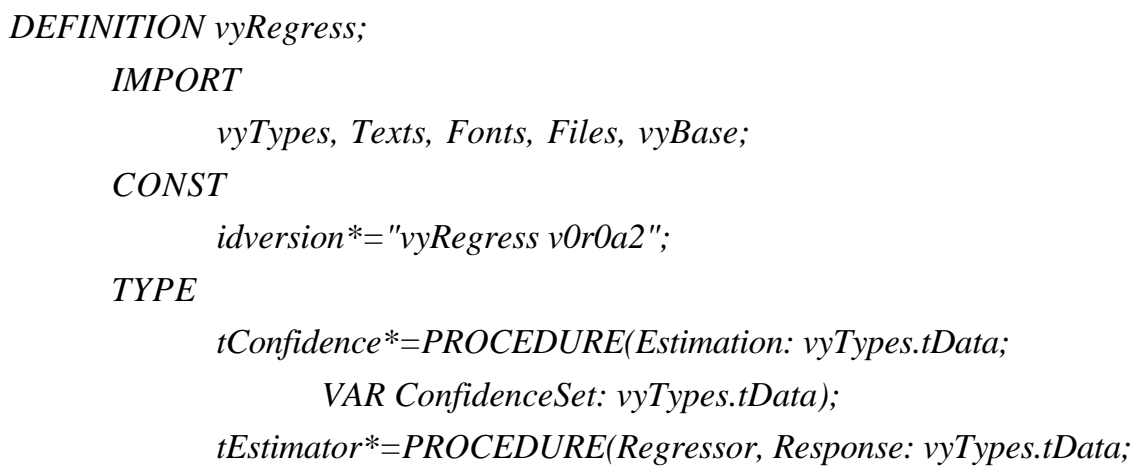

Once you have identified that "level" in the regression module may be the variable of interest for you, you can write a small program to modify it:

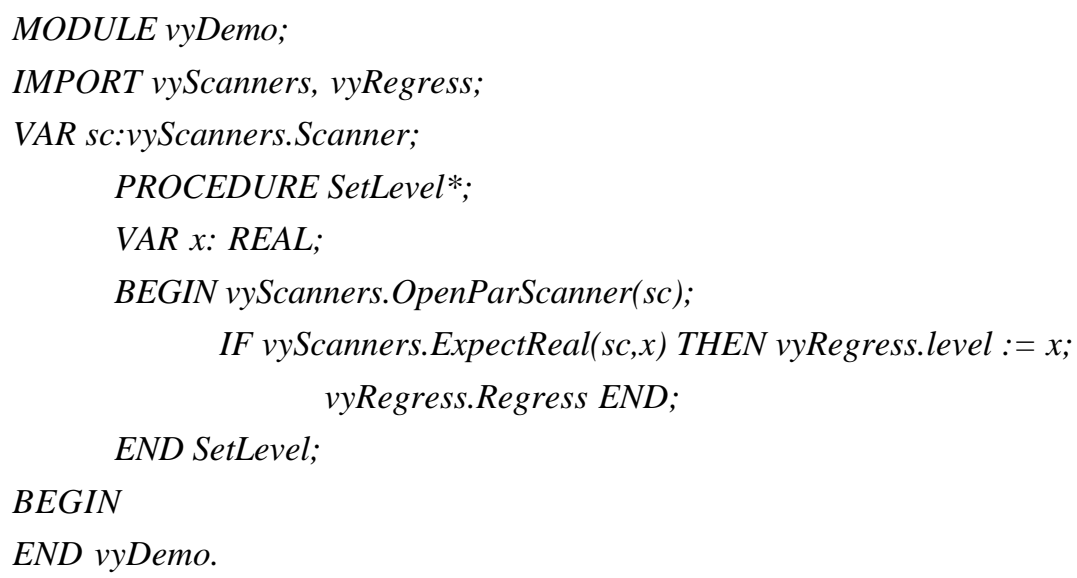

This is a small Oberon module which can be written and compiled even while the regression is up remember that once an Oberon module is loaded, it is persistent unless explicitely unloaded. So the regression module persists and all its information is preserved even while an editor or a compiler is invoked. The second line declares that the module may make use of services of other previously existing 
modules (vyTexts, In, vyRegress). [By convention, we use the prefix vy... for all Voyager modules at the current state of development]. The fourth line contains the declaration of a procedure, SetLevel. The star marks this procedure as exported, so it can be accessed from the outside. Since the procedure has no explicit parameters, by convention it defines a command which can be called by executing "vyDemo.SetLevel" in any text. It opens a scanner on the command text and tries to read a real number. If it succeeds, it uses the number to set a new level for the imported module vyRegress, writes a message reporting the new level, and redoes the regression. So the command SetLevel 0.99 may be just what you want. If you want more, you may want a slider to control the level. Grab into the bag of prepared components, take a slider and link it to the SetLevel command and you have taught the old regression program new tricks, namely to have confidence bands at a level which can be controlled dynamically by a slider.

If you want to have a different kind of simple regression, you can use SetEstimator to install a different regression algorithm. The Oberon type support covers procedures as first class members, and the Browser information shows the calling convention used for regression. If you supply a different regression algorithm, you can plug it in. You get the scatter plot for response versus regressor with overlaid regression curve and the residual plot for free. This is a generic display which comes with any simple regression. Of course you do not get confidence sets for free - the Scheffé confidence bands of the simple Gaussian model do not apply any longer, and you have to provide your own algorithm for confidence sets - or a NIL value if you don't have it. It would be risky to allow modification of the regression algorithm without adapting the confidence sets algorithm. Hence no direct access to these is allowed, but you are forced to use the installation procedure SetEstimator.

We have walked through this little examples in some detail to show how an extension can be made, using Voyager and Oberon. These are simple examples, but they should convey the idea. If you can augment a regression display to have controllable confidence bands, you get an idea what you can do in other situations. Since the state of the modules is preserved and memory is shared, extensions can access information from other modules, even if an extension is added at run time. Since the system is strictly modular, all previously available functionalities can be used in the extension.

As a more complex illustration, we use some examples from work in progress, communicated by M. Diller [Diller 1995], who is working on a system for the simulation of locally stationary time series using Voyager. Since all the components used here are extension of Voyager objects, and Voyager has knowledge of the dependency structure, we can use Voyager to get some insight in the structure of the simulation: Voyager can display the dependency structure as a list, or as a graph. [Figure 10]

$-16-$

30. Oct. 1995. Submitted to the Journal of Computational and Graphical Statistics WWW entry point: http://statlab.uni-heidelberg.de. Comments to gs@ statlab.uni-heidelberg.de, please. 
File Edit System Control :ww:Example.Log | Close. Copy Grow Segrch. Replace. Parcs Togls C N M Store. System.Log |Close Grow Locate Store !

This Frame should have an parc element of apropriate width.

asCmdX Insight.Show

\section{process generation}

The following commando generates a process

and returns the list of involved transformations.

WW.NewARMAProcess $\uparrow$ qrocessLength?

6412825651210248192 defaultSamplesize (= 512)

The following commando returns the selected transformation, modelled as a graph.

voyager.Show (a) as Insight $\sim$

Now, you can use the the buttons in the ines plot for futher investigations.

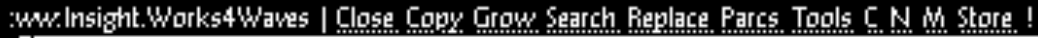

new process generation samplesize $=256$

TP0004B848

TImnovationsTP0004B848

TCoefficient TP0004B848

TÁRiWAPlot TP0004B848

Insight: TP0004B848

varianceFunctinOption nction IVariancenceOption livean eanOption generator leSize

WA Roots AR Roots

TCoefficient TP0004B848

$\longrightarrow$ ä Coefficient

mean function Wh Coefficient

TP0004B848

process prelude

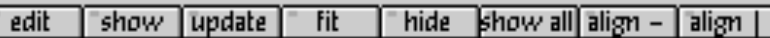

\section{System.Tool | Close Copy. Grow Search Store}

\section{gs.SoftStat System.Free Voyager: \\ Softstat.}

Edit.Open $\uparrow$

System.Showivodules

System.ShowCommands $\uparrow$

System.State $\uparrow$

Compiler.Compile *

Compiler.Compile $* / s \quad$ new sywbol file

Edit Free Dir. Dom Togls Mess w

ASCII.Write :gs:ASCII.Wod $=>: g s: A S C I I$. hod 1 m

:local:Local.Tool | Close Copy Grow Search Store

Ed.op Cmpos Cmpo Err Cmpt Sysop Dirt Diff

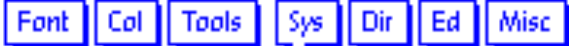

Ed.Sht Style Size Bigger Smaller.

G̈raphicElems. Insert Öberon.08.1. ̈̈raphn

System.Free wCmd:- ErrorElems.Wark $\uparrow$

Figure 10. Simulation of a (non-stationary) ARMA process using Voyager. The simulation is implemented as an extension of Voyager. Voyager can retrieve the dependency structure of the simulation components, and generated a dependency graph.

You can use a textual interface, or you can use this graph to inspect individual components. From the type of the component, Voyager ties to infere an appropriate representation for display. For example, selecting the "process" component and clicking "show" give a line plot of the simulated process.

[Figure 11]

-17 -

30. Oct. 1995. Submitted to the Journal of Computational and Graphical Statistics

WWW entry point: http://statlab.uni-heidelberg.de. Comments to gs@ statlab.uni-heidelberg.de, please. 
Figure 11. Simulation of an ARMA process using Voyager. Any component of the simulation, like the simulated process shown here, can be accessed from the component graph.

Some of the components refer to object types which can be edited. These are marked red in the dependency graph if you use a colour display. Components like the mean function over time are handled directly by Voyager: Voyager includes a graphical function editor which allows modify a function by commands, or by changing the function graph by just redrawing it using the mouse. Other components, like the roots used for the simulation, do not have a predefined editing action: the roots are best represented as a complex function evolving with time, and there is no predefined graphical editor for complex functions in Voyager. So the predefined real function editors are re-used and extended to allow a graphical specification of amplitude and phase of the roots. [Figure 12]

$-18-$

30. Oct. 1995. Submitted to the Journal of Computational and Graphical Statistics

WWW entry point: http://statlab.uni-heidelberg.de. Comments to gs@ statlab.uni-heidelberg.de, please. 
WWNEWARMAProcess $\uparrow$ processLengths

- $\quad 6412825651210248192$ default5amplesize (= 512)

The following commando returns the selected transformation, modelled as a graph.

Voyager.Show (a) as wwinsight $\sim$

Now, you can use the the buttons in the insight plot for futher investigations.

W.Newsample (a)

generates a new sample of the selected process and has exactly the same effect as the update

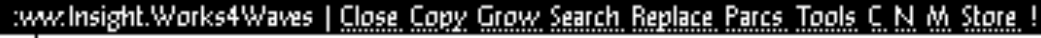

TARBWASPlot TP0004B848

Insight: TP0004B848

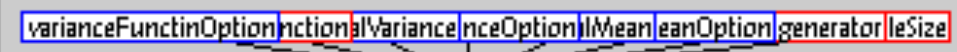
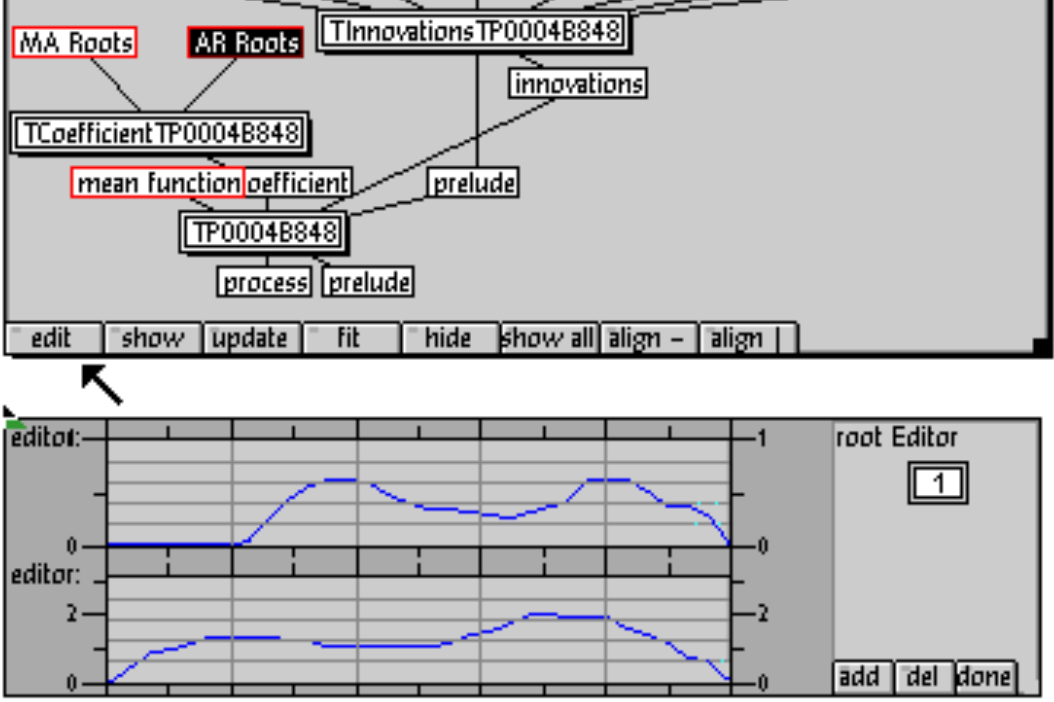

System.Tool | Cloge Copy. Grow Sejnech. Store
gs..Softstat. System.Free Voyager* *

Softstat.

Edit.Open $\uparrow$

Edit.Print QuickDraw*

System.Showhiodules

System.ShowCommands $\uparrow$

System.State $\uparrow$

Compiler.Compile *

Compiler.Compile $* / s \quad$ Hew syiwbol file

\section{:user:gs.Worksheet | Close Copy Grow Search Store}

Edit Free Dir. DomTogls Whess $W$

ÁSCil. Write :gs:ASCili.Mod $=>$ :gs:ASCII.Wod1 n

:local:Local.Tool | Cloge Copy Grow Segrch Stare

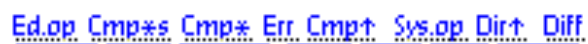

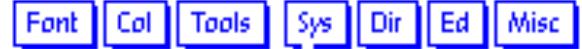

Ed.Sht style size Eigger smaller.

G̈raphicElems. Insert Öberon.08.1. ̈̈raphn

System.Free wCmd*a ErrorElems.Whark $\uparrow$

Figure 12. Graphical edition of the roots. Phase and amplitude of the ARMA coefficients used for the simulation can be modified using graphical mouse input: the predefined function editors are re-used and extended.

Voyager provides components designed for reusability and gives a rich reservoir of possibilities. The rôles have become clear: Oberon is a system providing the basis for extensible systems. And Oberon is changing the rules. It moves the focus from software libraries and closed systems to collections of software components and extensible systems. Voyager is laying a basis for applications in statistics, and provides the software components to build on.

\section{Behind the Scenes: Design Details}

The decomposition into components used here is quite different from conventional designs. We tried to follow the idea of separation of concerns, and we tried to remove unnecessary assumptions where possible. Voyager uses fairly abstract types as a base, and extends these types for specific applications.

-19 -

30. Oct. 1995. Submitted to the Journal of Computational and Graphical Statistics

WWW entry point: http://statlab.uni-heidelberg.de. Comments to gs@ statlab.uni-heidelberg.de, please. 
Although the design is object oriented, all computational routines are provided with a procedural interface to invite migration from conventional procedural models to Voyager: if you prefer classical procedural programming, you can use Voyager as a software library.

The Voyager object hierarchy is based on Items. An Item is a queuable object reference. It has an administrative slot, called next, which may be used to link to the next Item in a queue, and it has two informative slots, called $o$ (for object) and handle.The "o" field is used to reference an object, and the handle field is a generic slot to hold a message handling procedure. Items can be queued - various types of queue handling are supported and actions can be iterated in an abstract way throughout queues. Items are abstract objects. All functional semantics is to be defined by extensions. The Items type is defined to have a convenient common base for the administration of objects.

Everything which needs administration is implemented as an instance of Item. Everything which is worth an identity of its own is implemented as a Voyager Object. Voyager Objects are special cases of Items. As descendants, or sub-classes, of the Item class Voyager Objects support all queuing and housekeeping facilities by inheritance. Beyond this, Voyager Objects have an identity. They carry a unique identification mark, they have a creation and modification time, and they may have a name and a reference to dependent Object. The subscription mechanism described above is founded on the Voyager Object level. So it is possible to say "mark this Object as updated, and notify all other objects depending on this one". Elementary actions like "clone", "write yourself to a file", "load from file" are defined for all Object. However Objects, like items, are abstract classes. Functional semantics is still to be defined.

Usually, names are considered essential for the identification of objects. We have separated names from object identification. In Voyager, a name is but a user-defined reference to an object. Object identifications are handled by Voyager base, which has to guarantee unique identification. Names are handled by the names and command component, which has to do its best to resolve names and to find a corresponding object, possibly asking the user for more specific indications. It has to resolve possible ambiguities in a cooperative way and to inform the user if a name cannot be resolved reliably. But it may not try to control the user's preferences how to assign or use names. You can have various naming systems, even concurrently, operating on the same basic object management. This allows to have a generic naming system (such as using $\operatorname{Var}[5]$ ) along with various explicit naming systems (v, speed, speed $[\mathrm{km} / \mathrm{h}]$ ) for the same data. In particular, it is possible to "anonymize" data by using a randomized data base instead of the true name data base.

Data are special objects. The Data type is a descendant of the Voyager Objects type. It inherits all the administrative possibilities of Objects, and indirectly all the housekeeping facilities available for Items. Beyond this, logical and arithmetic operations are defined for Data. Following the Oberon conventions, this does not imply that Data actually execute this operations. A status field reflects any error code which may have occurred, such as "operation not implemented" or "type inappropriate". Data are abstract objects. One particular concrete extension is Vectors. Vectors hold an ordered list of entries (data) of one type. The public interface includes set/get operations for elements, and a variety of arithmetic operations. The storage representation of Vectors is kept internal, allowing for sparse representations, external storage and other variants. Data need not always be imported to some internal 
format, and sometimes may not even be on your local machine. A critical burden may be to assume that you know where your data are, and how they are stored. Assumptions of this kind should be avoided. Part of the data may be best represented by some generating rule (like for design matrices), or just by a reference on how to fetch the data if you really need them. The idea that all data are always present on your local machine as a table is obsolete - think of a distributed computing environment, where the data may come in from remote sources, possibly not synchronized.

A special component of Voyager is dedicated to the access and presentation of data. An obvious variant of this is a reader/writer which handles ASCII files with tables, but this is just one special variant. An agent cooperating with a data base management system may be a more natural replacement in other situations. Introducing data access as a separate component and delaying representation in a specific format as late as possible imposes some overhead, but it is prone to reveal some information which otherwise is easily hidden. For example, it was a natural consequence to introduce a scanner module which can be accessed directly: For the implementation of an ASCII reader/writer for example we had to make some scanner information accessible in a well defined way. The information comes in as patterns in an ASCII stream first. As a consequence, detailed information about the input stream is available from the scanner.

To illustrate the benefit, we take the well-known stack loss data set, which has been widely used as an illustration in robust statistics [Dodge 1994]. The data set has been useful to illustrate that the result of an analysis may critically depend on some few data cases (as well as on the choice of method). Most published analysis of this data set rely on a model with a continuous error distribution. The first information about this data set returned by Voyager is a mere reflection of the rectangular structure of the data file. Next, ahead of any statistical analysis, Voyager can give more detailed information about this data set. In this example, Voyager draws the attention to the fact that all variables - including the loss, which is the response in this example - are heavily discretized, and that the rate follows a pattern which suggests that a systematic experiment has taken place. For any data analysis, this is essential initial information which should be taken into account in the analysis. Any statistical system could supply this information, but in most systems considerable effort is necessary to get it. We do not know how many references to the stack loss data set have taken into account the simple fact that all values in this data set are strongly discretized or rounded. [Figure 13]

$-21-$

30. Oct. 1995. Submitted to the Journal of Computational and Graphical Statistics WWW entry point: http://statlab.uni-heidelberg.de. Comments to gs@ statlab.uni-heidelberg.de, please. 
* File Edit System control

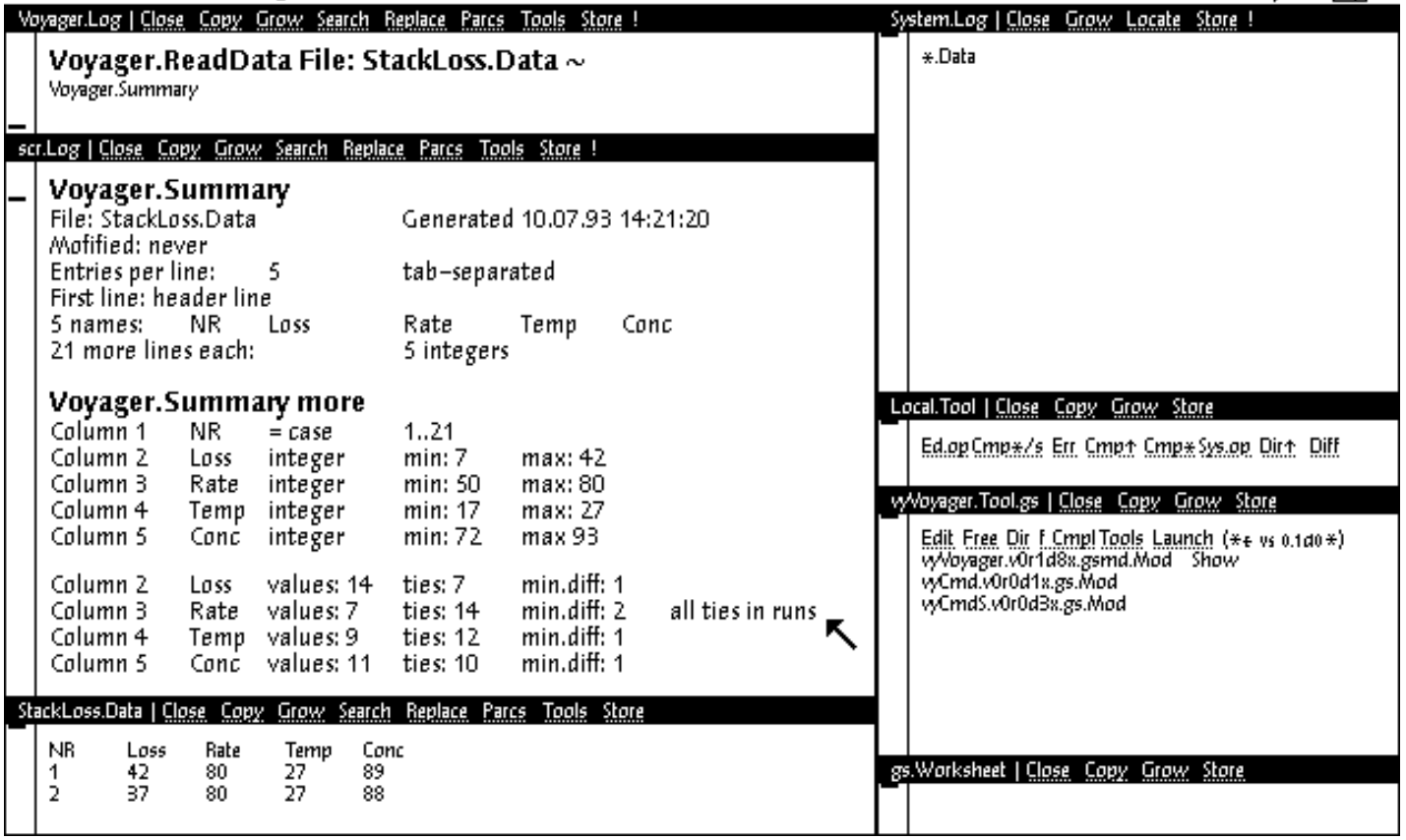

Figure 13. Information provided by the scanner. The Voyager scanner output provides detailed information which can be crucial to statistical analysis. Command Voyager.Summary gives information about the matrix structure of this data set. With one additional command Voyager.Summary more, Voyager reveals to the discrete structure of the data set, as seen from the high proportion of ties, and it points to the regular design of this experiment.

\section{Current State and Perspectives}

The role of statistical software is changing. Recent developments in software technologies allow thinking in terms of software components instead of systems. This brings us back to the old time of software libraries, but on a new level: the versatility of subroutine libraries can be maintained, but reliability and stability can be guaranteed on a system level. To use this, one needs a careful design, not only of single routines, but of components which can be implemented as well defined modules.

Following the usual Oberon conventions, the Voyager components have been implemented as a layered architecture. All components, as far as feasible, have a basic layer which contains abstract types and methods, and general services which are quiet, i.e. they do not require a user interface. Only the next layer gives a possibly visible layer with a concrete implementation. Access functions and interface routines requiring a specific user interface are collected in a third layer. So even if you do not want to make use of the Voyager system, you can use its algorithms as components. Application and interface programming can be decoupled, allowing for task specific interfaces while maintaining a consistent computational core.

Extensibility, even at run time, can be supported using all the type safety of a compiled system, and with all the performance benefits of compiled code. In an appropriate environment, like Oberon, this 
extensibility can be system wide, allowing a seamless integration of statistical software into a general computing environment.

Todays software technologies gives a basis to develop extensible and portable systems for statistics and data analysis. Reliability in an extensible environment is the major challenge. How this challenge can be met depends on the software tools and environment chosen in a first step. The choice of tools and environment is free first, but to guarantee stability and extensibility, the implementer has to pay afterwards for the choice taken. In an Oberon environment, system wide garbage collection, fine grained modularity and type safety across module boundary take care of many tasks which otherwise have to handled ad hoc. This gives a sound foundation for an extensible system.

\section{Acknowledgements}

Like all software products, Voyager is a result of many contributions. In particular, comments by R. Becker, B. Cleveland, L. Dümbgen, J. Marais, A. Unwin and A. Wilks have been helpful. Voyager has been inspired by ideas and examples seen in Data Desk [Velleman 1992], Regard [Unwin 1994], Diamond Fast [Unwin and Wills 1988] and in the Medas proposal [Biehler 1994]. The Voyager graphics system has been developed by M. Diller with support of F. Friedrich.

\section{Literature:}

Becker, R.A., Cleveland, W.S., Wilks, A.R. (1987), “Dynamic Graphics for Data Analysis,” (with discussion), Statistical Science, 2, 355-395.

Biehler, R. (1994), “Towards Requirements for More Adequate Software Tools That Support Both: Learning and Doing Statistics," presented at the Fourth International Conference on Teaching Statistics (ICOTS 4) Marrakech, Morocco, 25-30 July 1994. Manuscript. Bielefeld.

Diller, M. (1995), personal communication.

Dodge, Y (1995), “The Guinea Pig of Multiple Regression” in: H. Rieder (ed.). The Huber Festschrift.To Appear:. Springer: Heidelberg 1995

Franz, M. (1993), "Emulating an Operating System on Top of Another," Software-Practice and Experience, 23, 677-691.

Gutknecht, J. (1993), Oberon System 3 - A realm of Persistent Objects, preprint, ETH Zürich $<\mathrm{ftp}: / / \mathrm{ftp}$. inf.ethz.ch/pub/Oberon/System3>.

Knasmüller, M. (1994), Oberon Dialogs, technical report, Universität Linz.

Marais, J. (1994), “Oberon System 3,” Dr.Dobb's Journal, 220, 42-50.

Marais, J. (1995) The Gadgets Guide, ETH Zürich.

Marais, J. (1995a), "Extensible Software Systems in Oberon,” manuscript, ETH Zürich, submitted to -23 -

30. Oct. 1995. Submitted to the Journal of Computational and Graphical Statistics WWW entry point: http://statlab.uni-heidelberg.de. Comments to gs@ statlab.uni-heidelberg.de, please. 
$J C G S$

Mössenböck, H.; Wirth, N. (1991), “The Programming Language Oberon-2," Structured Programming, 12.

Pfister, C. et al. (1994), Oberon/F, Basel: Oberon microsystems.

Reiser, M. (1991), The Oberon System, Reading: Addison-Wesley.

Reiser, M.; Wirth, N. (1992), Programming in Oberon, Reading: Addison-Wesley.

Sawitzki, G. (1994), “A Portable, Object-Oriented Extensible Statistical Programming Environment based on Oberon," in: R. Dutter, W. Grossmann (eds.) Proceedings in Computational Statistics (Compstat 1994) pp. 158-159. Complete manuscript available as <file://statlab.uni-heidelberg.de/pub/Voyager/Compstat.ps> or <... .ps.gz>.

Sawitzki, G. (1994a), "Diagnostic Plots for One-Dimensional Data," in: P.Dirschedl \& R.Ostermann (eds.) Computational Statistics, Papers collected on the Occasion of the 25th Conference on Statistical Computing at Schloss Reisensburg. Heidelberg, Physica, 1994, ISBN 37908-0813-X, pp. 234-258.

Unwin, A.R., and Wills, G. (1988), "Eyeballing Time Series," Proceedings of the 1988 ASA Statistical Computing Section, 263-268.

Unwin, A.R. (1994), "REGARDing Geographic Data,” in: P.Dirschedl \& R.Ostermann (eds.) Computational Statistics, Papers collected on the Occasion of the 25th Conference on Statistical Computing at Schloss Reisensburg. Heidelberg, Physica, 1994, ISBN 37908-0813-X, 345-354.

Velleman, P.F. (1992), Data Desk, Ithaka New York: Data Description.

Wirth, N.; Gutknecht, J. (1989), "The Oberon System," Software-Practice and Experience, 19, 857-893.

Wirth, N.; Gutknecht, J. (1992), Project Oberon, Reading: Addison-Wesley.

Wirth, N. (1988), “The Programming Language Oberon," Software-Practice and Experience, 18, 671690 .

$-24-$

30. Oct. 1995. Submitted to the Journal of Computational and Graphical Statistics WWW entry point: http://statlab.uni-heidelberg.de. Comments to gs@ statlab.uni-heidelberg.de, please. 